\title{
A cloud-based fatigue analysis tool with interactive Neuber's master curve
}

\author{
Daniel Kujawski \\ Western Michigan University, Mech and Aerospace Eng., Kalamazoo, MI 49008-4353, USA
}

\begin{abstract}
A cloud-based fatigue analysis and life prediction tool is presented. Users around the globe may access it via Internet by means of multiple platforms such as desktop and laptop computers, tablets and/or smart-phones. It is an intuitive educational software, aimed also to assist designers in the pre-prototyping stage in fatigue life estimation for smooth or notched parts subjected to constant amplitude, block loading, and spectrum loading histories. For spectrum amplitude loading a dedicated spectrum software package is provided, which is essential for a potential clean-up and/or desired modifications of a raw spectrum data. Subsequently, a rainflow method is utilized and the corresponding hysteresis loops at the notch-root or critical "hot spot" location are determined and plotted. For notched components, an interactive Neuber's master curve is utilized and discussed. It is shown, that the Neubers's master curve is only material dependent and is applicable for both monotonic and cyclic loading situations.
\end{abstract}

\section{Introduction}

In general, fatigue damage occurs in materials when subjected to variable amplitude stresses that are below the ultimate tensile strength, or even yield strength of the material [1]. Components are designed to an optimal safety factor; one high enough to avoid failure by fatigue, but low enough to avoid overly-bulky components and consequently excessive cost and material waste. Typically, Research \& Development (R\&D) departments employ highly trained engineers to perform fatigue analysis on components before and during a prototype development. This involves extensive time and resources to create new designs. Due to cut off in R\&D budgets many new designs are based on old and proven designs.

In the global and highly competitive market, the R\&D departments are mostly afforded by relatively large organizations. These $R \& D$ departments routinely acquire dedicated fatigue/durability software, which are accompanied often with relatively expensive annual license fees. On the other hand, smaller organizations rather seldom have R\&D departments but they may have a periodic need for fatigue/durability analysis. According to Forbes (2016) many engineering graduates don't have design confidence and know how, in particular in durability design and fatigue analysis. Figure 1 illustrates an overall need for a conducive fatigue/durability software, which would bridge theory thought in classroom with industry application. Such software should be:

-easy to use,

-interactive with step-by-step approach, 
-informative with basic explanations of background theory,

-cloud-based: accessible anytime and on any devise.

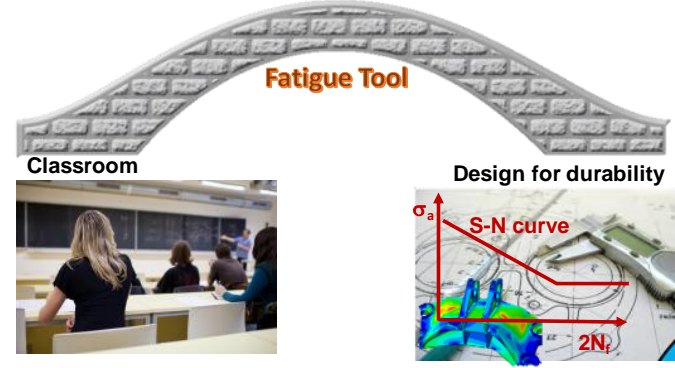

Fig. 1 Bridging theory with idustry application.

Thus, there is an overall demand by small and medium manufactures for a straightforward web-based fatigue analysis tool, which can be accessed via any internet connected device on as needed basis. In an age of increased technological mobility, there lies opportunity to create tools which are easily accessible to students outside of the traditional classroom environment. This 'elearning' trend among students in colleges is growing exponentially.

In order to carry out a meaningful fatigue analysis by new graduates it requires some fundamental knowledge, which most students would not have learned during their undergraduate studies. Also, most practices in small companies are limited when it comes to fatigue analysis and life prediction know how experiences. Currently, there exist some free web-based fatigue analysis tools [e.g. 2,3], which include fatigue calculators, material databases and allow to perform limited fatigue analysis on the Web. However, both of these sites have limited interactive and self-explanatory capabilities.

The aim of this paper is twofold. Firstly, it communicates a fundamental knowledge associated with popular fatigue life prediction approaches. Secondly, it presents a novel interactive web-based tool for learning a modern fatigue analysis and life prediction methodology of smooth and notched components. The web-based tool may be easily accessed by means of multiple platforms such as desktop and laptop computers, tablets

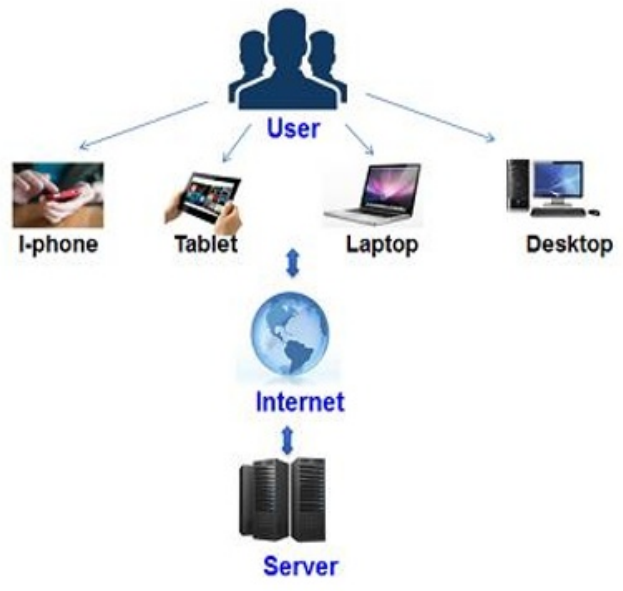

Fig. 2 Illustration of the web-based access. and/or smart-phones as it is illustrated in Fig. 2. A user with various degree of fatigue background would benefit from the proposed interactive "on-the-fly" learning experience. At each step, concise fundamental fatigue information is provided without the need of a tutorial handbook. It constitutes interactive, selfexplanatory web-based educational tool, which allows the users to learn the fatigue fundamentals as well as to expand and master their knowledge on modern fatigue analysis methods. For variable amplitude loading histories a rainflow method is used to count the cycles and to determine the corresponding hysteresis loops at the critical locations. In addition, a dedicated spectrum analysis tool is provided which enables clean-up and desired modifications of a raw spectrum data. During analysis the relevant interactive graphs and calculated values are displayed. The following sections provide a summary of main features of the proposed web-based interactive fatigue analysis learning tool. 


\section{Web-based fatigue tool}

The website is structured to be simple and self-explanatory, flowing from Step 1 to Step 4, as it is discussed in this section.

The current website features of this educational version provides basic explanations and video examples how to use the software. The website also provides additional spectrum analysis software to prime the raw spectrum data before fatigue life prediction analysis can be carried out using the educational version. In this section, the analysis procedure is discussed with different options available on the website. As the user enters the website, the home page is presented. Under 'Fatigue Basics', the 'software' tab is selected which presents the life predication software. Below are the steps involved which are illustrated with screenshots of Steps 1 to 4 . This version of the software works best with Chrom browser.

\section{Step 1: Select Material}

In this step, the desired material and units are selected. Figure 3 depicts a screenshot related to Step1. The educational version only provides five materials with an additional feature to input user defined material properties.

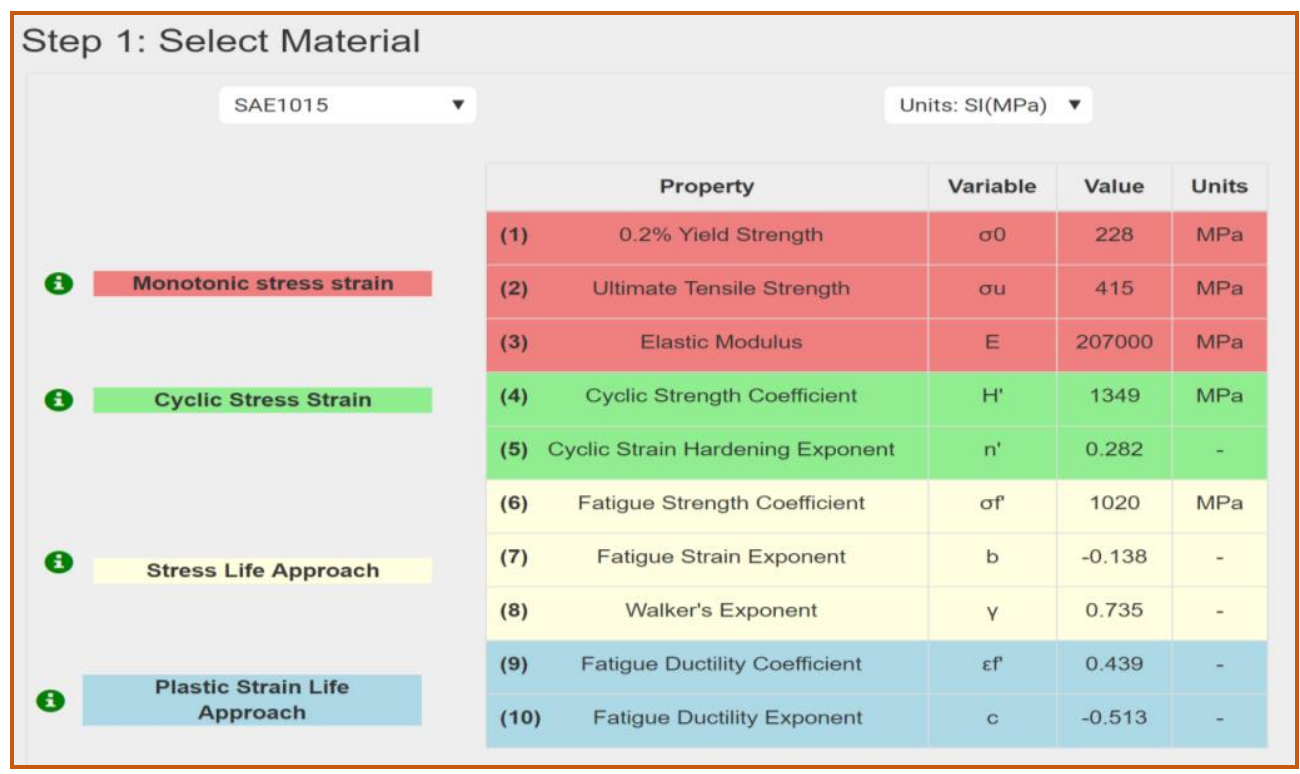

Fig. 3 Screenshot of the Step1.

Materials properties are arranged in four groups related to:

-Monotonic stress-strain,

-Cyclic stress-strain,

-Stress life approach,

-Plastic strain life approach

By clicking on an icon (i) the user would be presented an additional window, shown in Fig. 4, with helpful tips and information for the associated properties. To facilitate the identification of these properties, numbers from 1 to 10 are assigned to each individual property, that can be easely found in Fig. 4 regarding their definition and determination. 

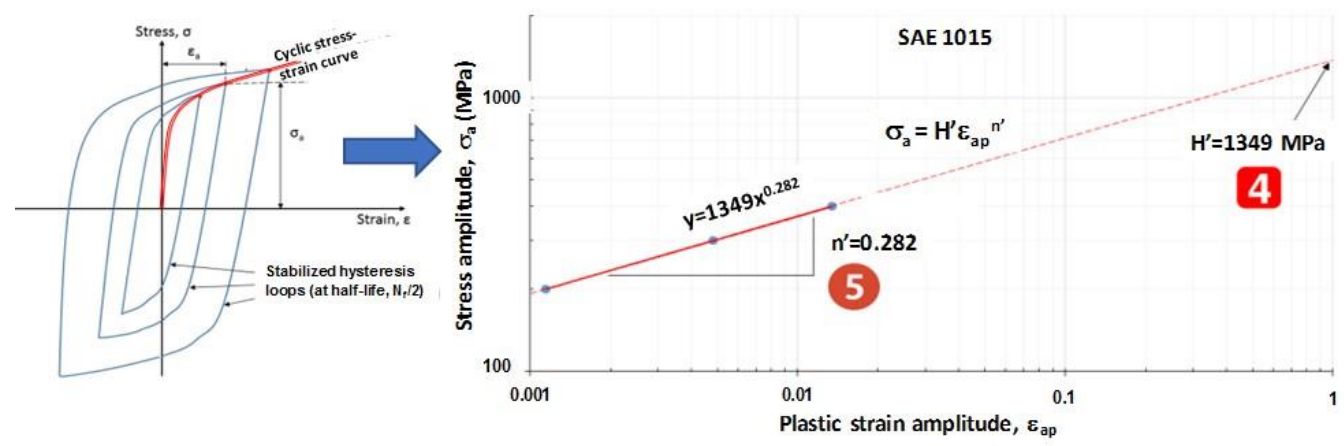

Fig. 4 Graphical illustration related to determination of the cyclic stress-strain curve.

For example, the cyclic stress-strain has two properties H' and n' designated by numbers 4 and 5, respectively. The cyclic stress-strain curve is obtained by connecting the tips of stabilized hysteresis loops (corresponding to half-life, $\mathrm{N}_{\mathrm{f}} / 2$ ) attained under symmetric strain-controlled tests, as it is illustrated on the left graph in Fig. 4. Each stabilized hysteresis loop corresponds to fully-reversed strain-controlled test at a specified value of the strain amplitude. Typically, multiple specimens are used where one specimen is utilized to obtain a stabilized hysteresis loop. Determination of the constants $\mathrm{H}^{\prime}$ and n' from log-log plot is illustrated on the right graph in Fig.4.

\section{Step 2: Specimen}

In this step, the users are provided with an option to choose a smooth or a notched specimen/component for analysis. Selecting a smooth specimen would result in a stress concentration $\mathrm{k}_{\mathrm{t}}$ value of 1 to be used in the analysis, which the users cannot change. While for a notched specimen the users would be able to input the desired $k_{t}$ value $\left(k_{t}=2\right.$ is default) or fatigue notch factor $\mathrm{k}_{\mathrm{f}}$ in the proceeding step.

\section{Step 2: Specimen}

$\checkmark$

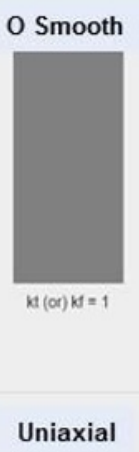

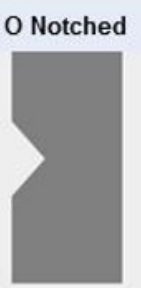

Multiaxial
(1)

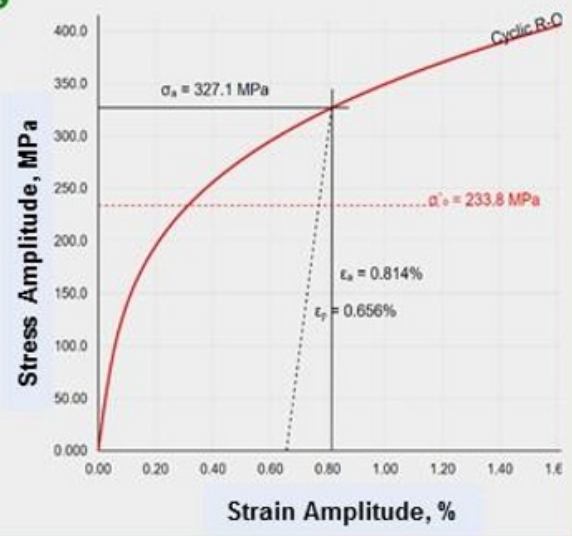

Fig. 5 Screenshot of the Step 2 showing specimen types and the Ramberg-Osgood (R-O) curve. 
The values of $\mathrm{k}_{\mathrm{t}}$ (or $\mathrm{k}_{\mathrm{f}}$ ) are used in analysis of notched components in tandem with nominal stresses. The default value is 2 but may be changed according to the users need.

A smooth specimen is a placeholder representing smooth component or notched component for which elastic-plastic stress and strain are known (e.g. obtain from an elasticplastic FEA calculations). If smooth specimen is selected in Step 2, users would have the option to select either stress or strain as the input data type. On the other hand, a notched specimen is a placeholder for component with notches, which is analyzed using a nominal stress or an elastic stress from linear-elastic FEA calculations. If the input data corresponds to linear-elastic stresses from FEA than the stress conentration factor, $\mathrm{k}_{\mathrm{t}}=1$ is utilized. An analysis of notched component, which utilizes nominal or elastic FEA stresses require plasticity correction using Neuber's rule [4] and is discussed in section 3.1 in Step 3.

\section{Step 3 : Loading Condition}

The user can select three loading type to be analyzed as it shown in Fig. 6. The software is designed to analyze the following loading types:

1. Constant Amplitude

2. Block Loading

3. Spectrum Loading

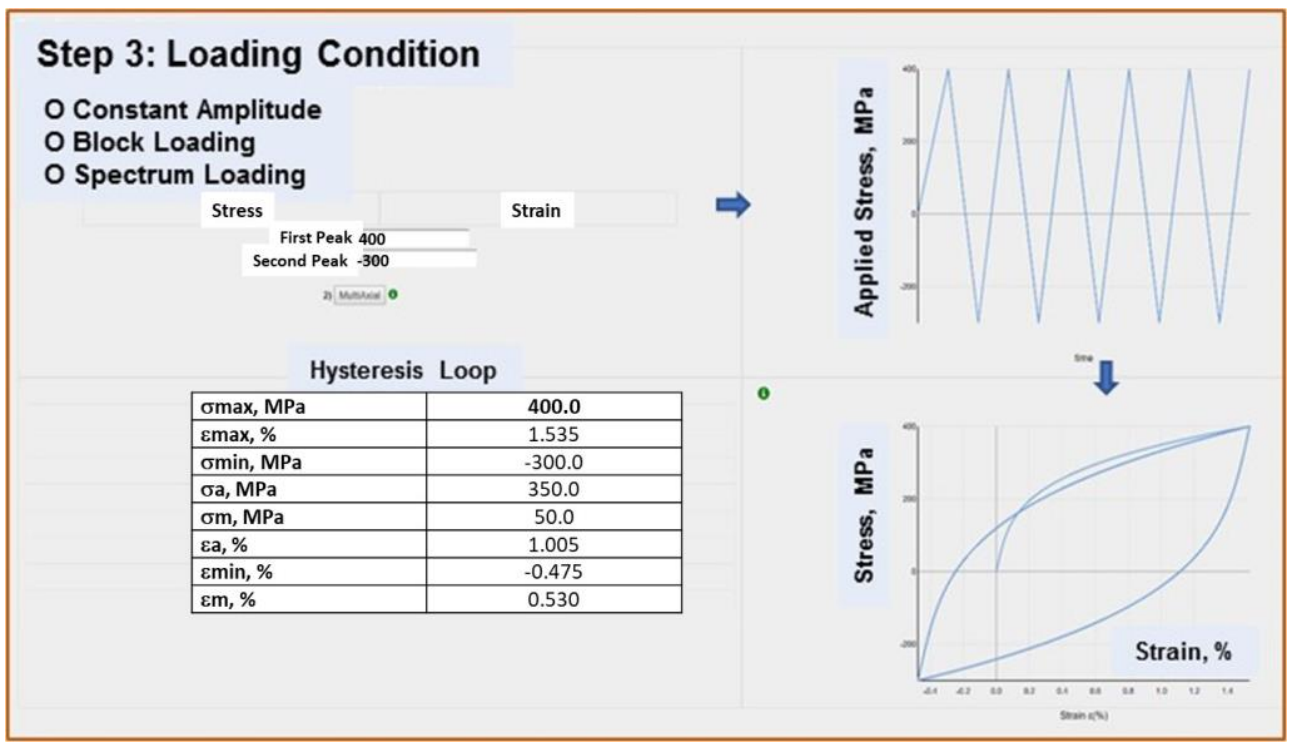

Fig. 6 Screenshot of the Step 3 for a constant amplitude loading.

For a Constant Amplitude loading the user shall specify the loading levels as 'First Peak' and 'Second Peak'. Loading always starts at zero and continue towards to 'First Peak', which can be the maximum or the minimum stress. A Block Loading can be inputed using: a file, table data, or easy table. A Spectrum Loading can be inputed in form of the text file where peaks and vallys are listed in sequenctial order. Proper information is displayed if the user chooses Block Loading or Spectrum Loading options.

For each loading type, the graph of applied loading and corresponding hysteresis loops are displied, as it is depicted on Fig.7. In addition, the output window, provides a consolidated numerical values for maximum, minimum, mean and amplitude of stress and strain at the notch tip for the largest hysteresis loop. 
The shape of the hysteresis loop branches corresponds to the doubled cyclic stress-strain curve called Masing model [5]. Masing and memory rules are utilized to appropriately

(a) Applied Loading

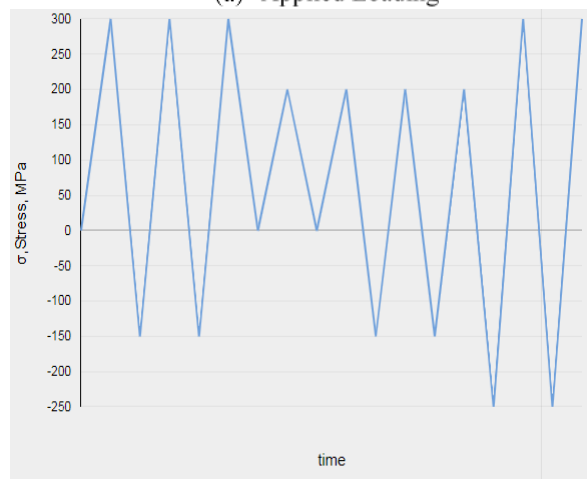

(b) Hysteresis Loops

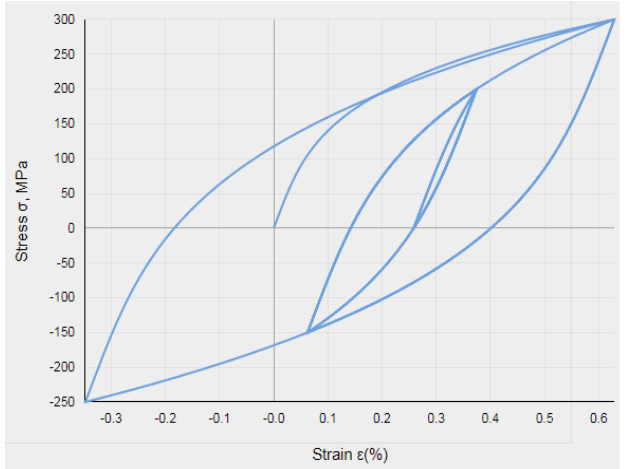

Fig. 7 Screenshot illustrating applied variable loading and corresponding hysteresis loops.

define a damage cycles from closed hysteresis loops, so that it can be considered in both drawing and the well known Palmgren-Miner fatigue damage analysis.

For a block or spectrum load history, the stress-strain hysteresis loops exhibit three memory rules (MR) that can be summarized as:

-MR-1 : After forming a closed hysteresis loop, the starting point of which has been on the cyclic stress-strain curve, the stress-strain path followsthe cyclic stress-strain curve.

-MR-2 : After forming a closed hysteresis loop, the starting point of which has been on a superior hysteresis loop, the stress-strain path follows the orginal hysteresis loop.

-MR-3 : A hysteresis loop started on the cyclic stress-strain curve ends in the opposite quadrant when the stress or strain amount of its starting pointis reached. Subsequent loading follows the cyclic stress-strain curve.

\section{1 : Interactive Neuber's curve}

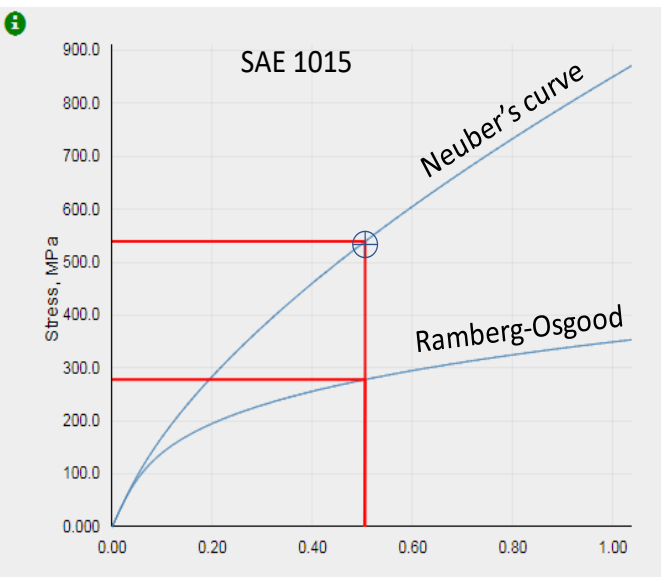

Fig. 8 Screenshot of the interactive Neuber's curve.
Selecting a notched specimen in Step 2 the graph displayes both the RambergOsgood curve and an interactive Neuber's curve [6] for a selected material as it is depicted in Fig. 8. The interactive master Neuber's curve is a core of this notch stress/strain analysis software. It can be noted, that the Neubers's master curve is only material dependent and is applicable for both monotonic and cyclic loading situations. The Neuber's master curve represents the values of the theoretical elastic stresses and the actual elastic-plastic strain at the notch root (or 'hot spot'). Thus, the crosssection of a vertical line with the RambergOsgood curve determines the estimated elastic-plastic stress and strain at the notch root or 'hot spot' location. 


\section{Step 4 : Life Prediction}

In this step one of the three life prediction method ban be selected, i.e.

-stress-based [7], or

-strain-based [8,9], or

-stress/strain-based [10].

In the stress-based approach the mean-stress effect is modeled according to widely used Goodman [11], Morrow [8], SWT [10], and Walker [12] approaches. For constant amplitude loading a number of reversals to failure is provided for each mean stress approach model. In addition, the fatigue limit corresponding to fully-reversed cycling is also presented as it is illustrated by the last column in Fig. 9. shown.

For block or spectrum loading a number of repetition to failure is calculated and

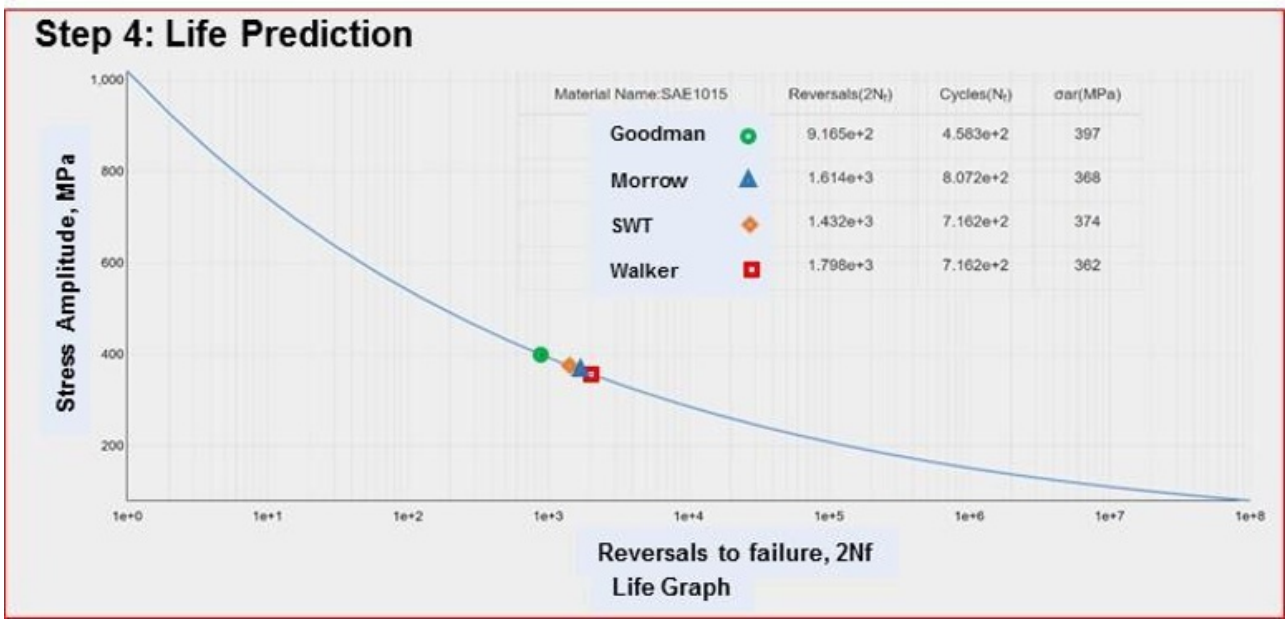

Fig. 9 Screenshot of the stress-based life graph.

After completion of the analysis, a report can be printed summarizing all 4 steps or only selected steps can be chosen for printing.

\section{Conclusions}

An interactive web-based tool for a modern fatigue analysis and life prediction methodology of smooth and notched components has been developed. Users from around the globe with diverse degree of fatigue knowledge may access it via Internet by means of multiple platforms such as desktop and laptop computers, tablets and/or smart-phones. In particular, the users with a limited fatigue background would benefit from possibility to expand their fatigue learning experience. The tool can be used to analyze constant amplitude, block loading and spectrum loading histories. Dedicated spectrum analysis software is also included. Life predictive capabilities include stress-based, strain- based and stress/strain-based approaches including mean stress effects. 


\section{Acknowledgements}

This research is supported by Western Michigan University Technology Development Fund and Durabilika, LLC.

\section{References}

1. N.E. Dowling, Mechanical Behavior of Materials, fourth ed., Prentice Hall, Upper Saddle River, New Jersey (2013)

2. https://www.efatigue.com

3. http://fde.uwaterloo

4. H. Neuber, Theory of stress concentration for shear strained prismatic bodies with arbitrary non-linear stress strain law, J. Appl. Mechanics, Dec.:544-550 (1961)

5. G. Masing, Eigenspannungen und Verfestigung beim Messing, in Proc. of $2^{\text {nd }}$ Int. Congress of Appl. Mech., Zurich (1926)

6. D. Kujawski, J.L.K. Teo, A generalization of Neuber's rule for numerical applications, Procedia Structural Integrity 5 : 883-888 (2017)

7. O.H Basquin, The exponential law of endurance tests. Proc Am Soc Test Mater. 10 : 625-30 (1919)

8. J.D. Morrow, Fatigue properties of metals. In: Fatigue design handbook Sec. 3.2. SAE Advances in Engineering, 21-30 (1968)

9. L.F. Coffin, A study of the effect of cyclic thermal stresses on a ductile metal. Trans ASME 76 : 931-50 (1954)

10. K.N. Smith, P. Watson, T.H. Topper, A stress-strain functions for the fatigue of metals. J Mater. $8: 767-78$ (1970)

11. J. Goodman, Mechanics Applied to Engineering. London: Longmans Green, (1899) [see also, 1, 9th ed., (1930)].

12. K. Walker, The effects of stress ratio during crack propagation and fatigue for 2024-T3 and 7075-T6 aluminum, Effects of Environment and Complex Load History on Fatigue Life, ASTM STP 462, Am. Soc. for Testing and Materials, West Conshohocken, PA, 1-14 (1970) 